\title{
Scaling-up voluntary medical male circumcision - what have we learned?
}

This article was published in the following Dove Press journal:

HIVIAIDS - Research and Palliative Care

8 October 2014

Number of times this article has been viewed

\author{
Jenny H Ledikwe ${ }^{1,2, *}$ \\ Robert O Nyangal,* \\ Jaclyn Hagon ${ }^{2}$ \\ Jessica S Grignon ${ }^{1,2}$ \\ Mulamuli Mpofu' \\ Bazghina-werq Semo ${ }^{1,2}$ \\ 'International Training and Education \\ Center for Health, Botswana, \\ Gaborone, Botswana; ${ }^{2}$ Department \\ of Global Health, University of \\ Washington, Seattle, WA, USA \\ *These authors are joint first authors
}

Video abstract

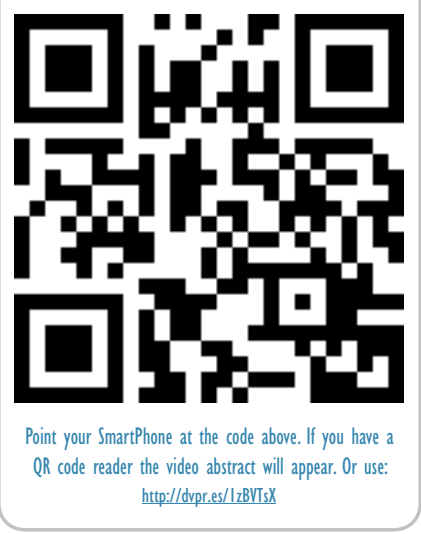

Correspondence: Bazghina-werq Semo PO Box AC46, ACH, Riverwalk,

Gaborone, Botswana

Tel +2673900925

Fax +2673900952

Email bazsemo@uw.edu
Abstract: In 2007, the World Health Organization (WHO) and the joint United Nations agency program on HIV/AIDS (UNAIDS) recommended voluntary medical male circumcision (VMMC) as an add-on strategy for HIV prevention. Fourteen priority countries were tasked with scaling-up VMMC services to $80 \%$ of HIV-negative men aged $15-49$ years by 2016 , representing a combined target of 20 million circumcisions. By December 2012, approximately 3 million procedures had been conducted. Within the following year, there was marked improvement in the pace of the scale-up. During 2013, the total number of circumcisions performed nearly doubled, with approximately 6 million total circumcisions conducted by the end of the year, reaching $30 \%$ of the initial target. The purpose of this review article was to apply a systems thinking approach, using the WHO health systems building blocks as a framework to examine the factors influencing the scale-up of the VMMC programs from 2008-2013. Facilitators that accelerated the VMMC program scale-up included: country ownership; sustained political will; service delivery efficiencies, such as task shifting and task sharing; use of outreach and mobile services; disposable, prepackaged VMMC kits; external funding; and a standardized set of indicators for VMMC. A low demand for the procedure has been a major barrier to achieving circumcision targets, while weak supply chain management systems and the lack of adequate financial resources with a heavy reliance on donor support have also adversely affected scale-up. Health systems strengthening initiatives and innovations have progressively improved VMMC service delivery, but an understanding of the contextual barriers and the facilitators of demand for the procedure is critical in reaching targets. There is a need for countries implementing VMMC programs to share their experiences more frequently to identify and to enhance best practices by other programs.

Keywords: voluntary medical male circumcision, HIV prevention, health systems strengthening, Africa, facilitators, barriers

\section{Introduction}

In 2007, the World Health Organization (WHO) and the joint United Nations program on HIV/AIDS (UNAIDS) recommended voluntary medical male circumcision (VMMC) as an add-on strategy for HIV prevention, ${ }^{1}$ based on the evidence from three randomized clinical trials demonstrating that VMMC reduces the risk of acquiring HIV infection by $51 \%-60 \%$ among heterosexual men..$^{2-4}$ Subsequently, 14 priority countries in eastern and southern Africa with high HIV prevalence rates and low levels of male circumcision began following recommendations to scale-up VMMC services to $80 \%$ of HIV-negative men aged 15-49 years by $2016 .{ }^{5}$ This generated a combined target of 20 million circumcisions. 5 
As presented in Table 1, the WHO reported that 3,158,556 procedures were conducted by December 2012, representing $15 \%$ of the overall target. ${ }^{5}$ There was, however, considerable variation across the priority countries. Kenya and Ethiopia made significant progress. These countries reported circumcising $63 \%$ and $57 \%$ of their target populations, respectively, while the remaining 12 priority countries achieved $<30 \%$ of their target. ${ }^{5}$ In 2013 , there was a marked improvement in the pace of scale-up with close to 3 million procedures having been reported. ${ }^{6}$ This included 2,270,200 procedures performed in sites supported by the US President's Emergency Plan for AIDS Relief (PEPFAR). ${ }^{7}$ While the total number of circumcisions conducted doubled during 2013, reaching a cumulative total of approximately 6 million, this only represents close to $30 \%$ of the initial target coverage level in the priority countries. ${ }^{6}$

The purpose of this review article is to apply a systems thinking approach, using the WHO health systems building blocks to explore factors that influenced the scale-up of the national VMMC programs from 2008-2013. Systems thinking is a WHO-recommended approach to problem solving that involves a broad examination of the health system. ${ }^{8}$ It is particularly recommended for complex interventions, such as the scale-up of national programs. ${ }^{8}$ The WHO health systems building blocks, which define the essential components of a health system, ${ }^{9}$ allow for a comprehensive examination of the

Table I Number of VMMC procedures conducted by the priority countries

\begin{tabular}{llll}
\hline Country & $\begin{array}{l}\text { VMMC procedures } \\
\text { done (2008-20I2) }\end{array}$ & Target & \% of target \\
\hline Botswana & 63,863 & 345,244 & $18.5 \%$ \\
Ethiopia & 22,961 & 40,000 & $57.4 \%$ \\
Kenya & 543,000 & 860,000 & $63.1 \%$ \\
Lesotho & 10,521 & 376,795 & $2.8 \%$ \\
Malawi & 36,250 & $2,101,556$ & $1.7 \%$ \\
Mozambique & 172,325 & $1,059,104$ & $16.3 \%$ \\
Namibia & 12,973 & 330,218 & $3.9 \%$ \\
Rwanda & 165,405 & $1,746,052$ & $9.5 \%$ \\
South Africa & 864,210 & $4,333,134$ & $19.9 \%$ \\
Swaziland & 48,083 & 183,450 & $26.2 \%$ \\
Tanzania & 319,320 & $1,373,271$ & $23.3 \%$ \\
Uganda & 467,318 & $4,245,184$ & $11.0 \%$ \\
Zambia & 340,992 & $1,949,292$ & $17.5 \%$ \\
Zimbabwe & 91,335 & $1,912,595$ & $4.8 \%$ \\
Total & $\mathbf{3 , 1 5 8 , 5 5 6}$ & $\mathbf{2 0 , 8 5 5 , 9 0 5}$ & $\mathbf{1 5 . 1} \%$ \\
\hline
\end{tabular}

Note: Reproduced with the permission of the publisher, from the World Health Organization (WHO) Progress Brief - Voluntary medical male circumcision for HIV prevention in priority countries of East and Southern Africa. July 2014. Available from: http://www.who.int/hiv/topics/malecircumcision/male-circumcision-info-2014l enl. Accessed May 15, 2014.

Abbreviations: VMMC, voluntary medical male circumcision; WHO, World Health Organization; HIV, human immunodeficiency virus.
VMMC program scale-up. The six building blocks include: 1) leadership and governance; 2) health workforce; 3 ) health service delivery; 4) medical products, vaccines, and technologies; 5) health financing; and 6) health information. This review article analyzes and summarizes key findings from peerreviewed publications, donor and technical agency reports.

\section{Leadership and governance}

Sustained support at global and national levels, as well as at community and facility levels, is key factor for successful VMMC scale-up

Key success factors related to leadership and governance include sustained country ownership and political will. The more successful countries, those such as Kenya which made significant progress toward meeting circumcision targets, maintained ownership of the VMMC program implementation and sustained political support at all levels of government throughout the entirety of the implementation. Examples of high-level country ownership and political will include engaging national champions, developing national policy and operational plans, designating a national program focal person, and conducting pilots with government involvement. ${ }^{10,11}$ It is, however, also important to ensure engagement at the community and facility level through the designation of the regional, subregional, and facility focal persons. The VMMC program achievements in Kenya have been partially attributed to not only the initial political support, but also to the continued commitment and engagement of the key stakeholders, including community members, implementing partners, politicians, and religious leaders. ${ }^{10-14}$ For example, the sustained community engagement of the Luo Council of Elders, the former Kenyan prime minister, politicians, and other stakeholders provided critical leadership in demonstrating that VMMC was medically beneficial and did not contradict political, cultural, or religious beliefs. ${ }^{12,14}$

It is important to note that the high-level support for VMMC programs, while important, has not necessarily translated into VMMC program achievements. ${ }^{11}$ For example, 11 of the 14 priority countries had developed national VMMC strategies and operations plans within three years of the WHO/UNAIDS recommendation to scale-up VMMC,,$^{6,10,11}$ but most of these countries are still far from reaching their targets. At the subnational level, the VMMC leadership has often been led by donor-funded international partners, which can minimize program ownership at the local level. ${ }^{5}$ 


\section{Health workforce}

Efficiencies to minimize human resource constraints are critical for scale-up

The ambitious, time-sensitive VMMC targets require large numbers of trained health care workers and other cadres of support personnel. ${ }^{15}$ An important facilitator of program scale-up has been the implementation of various surgical efficiencies outlined for consideration in WHO-guidance related to models for optimizing volume and efficiency. ${ }^{16}$ The models for optimizing volume and efficiency include a set of innovative service delivery strategies for improving human resource efficiency, such as task shifting, task sharing, adjustment of the ratio of physicians/primary providers to nurses, optimizing facility space through rotation among multiple surgical bays, use of electrocautery, and use of the forceps-guided surgical method. ${ }^{16}$

With the realization that physicians alone would be unable to meet the human resource needs for VMMC service provision during the scale-up phase, some countries introduced task shifting and task sharing. Task shifting involves the complete delegation of all the steps of a circumcision procedure to nonphysician or lower cadres of health care workers, while task sharing involves allowing lower cadres of providers to perform certain steps of the VMMC procedures, such as administering anesthesia and completing suturing. ${ }^{17}$ A systematic review and meta-analysis of ten research studies have shown that with the appropriate training, the task shifting of VMMC to nonphysician clinicians is safe and does not compromise the quality of care and services. ${ }^{18,19}$

By the end of 2012, eight of the 14 priority countries had implemented policies that allowed nonphysician clinicians to perform VMMC. ${ }^{5}$ Following the institution of VMMC task shifting in Kenya, two 30-day campaigns were conducted in which a total of more than 90,000 circumcisions were performed, more than likely would have been achieved if the program relied on physicians to conduct the procedures. ${ }^{20}$

Innovative surgical room organizations were tested in the Bophelo Pele project in Orange Farm, South Africa, demonstrating that task sharing combined with the use of multiple surgical bays optimized the number of patients that could be attended in high-volume settings. ${ }^{21}$ The rotation of health workers among multiple surgical bays is a widespread practice in Zimbabwe, Tanzania, and South Africa. ${ }^{17}$ Further research is warranted to understand better how these efficiencies can be applied in the most efficient and effective manner.
While task shifting, task sharing, the use of multiple surgical bays, and other efficiencies can have a positive impact on the VMMC program implementation, it is important to explore how other aspects of the health system may be affected. For example, recent data suggest this has resulted in some levels of fatigue and burnout of the health workforce. ${ }^{22}$ Consideration of work fatigue and burnout as VMMC scale-up intensifies is critical in preventing provider attrition and maximizing performance. It is also important that efficiency considerations be contextualized appropriately in different settings.

Data from Kenya suggest that task sharing was not wellreceived by the practitioners due to a belief that the provider has a professional obligation to remain with one client from the start to the end of the procedure. ${ }^{17} \mathrm{~A}$ quality-of-care assessment in South Africa suggests that the rapid scale-up of the VMMC program has diluted human resources and had an adverse impact on health care delivery with declines in the provision of quality services. ${ }^{23}$ With the expansion of services, it is critical to monitor the quality of care and services and to implement quality improvement initiatives to realize the full impact of VMMC on HIV prevention. ${ }^{24}$ This highlights the importance of taking a broad health systems perspective when examining VMMC programs.

\section{Health service delivery}

Expanding access and improving demand for VMMC are essential to service utilization

Key success factors include supplementing service delivery at fixed/static sites with other modalities of service provision, including outreach and mobile VMMC services to expand access. Outreach services encompass the delivery of periodic VMMC at health facilities that do not provide the services on a regular basis, while mobile services are delivered at nonhealth care facilities. Creative mobile and outreach initiatives involving the use of tents, mobile trucks, and prefabricated clinics have expanded access to the VMMC services while simultaneously addressing surgical space challenges at health facilities. ${ }^{25}$

In addition to varying the modalities of service delivery, efforts to modify the intensity of service delivery have also been beneficial to program implementation. Targeted campaign-style outreaches and mobile services have been used successfully during specific times of the year, such as during school holidays and the end of the harvest season. ${ }^{20}$

The Rapid Results Initiative in Kenya has been successful in meeting demand using mobile, rapid-response teams that 
are quickly deployed to areas of high demand. ${ }^{26}$ Promising data from a recent econometric analysis suggest that the VMMC campaigns can increase patient volume, reduce cost, and achieve efficiencies in economies of scale. ${ }^{27}$

While several successes have been documented through the various service delivery modalities and intensities described above, the recently published literature from Tanzania and Zimbabwe has shown the importance of formative research on community preferences in the design of service delivery modalities and intensity (routine versus campaign), as these affect the profile of the clients accessing services. ${ }^{28}$ This highlights the importance of targeting demand creation activities to specific segments of the population. To date, the majority of individuals participating in VMMC programs have been under the age of 25 , even though the programs aim to reach men aged $15-49 .{ }^{6}$ Demand creation messages need to be tailored particularly to those populations most at risk of HIV infection. Understanding barriers and motivating factors related to VMMC uptake is critical. For example, data from Tanzania suggest that younger individuals may be greatly influenced by their peers; ${ }^{28}$ whereas, data from Zimbabwe suggest older individuals may be more influenced by improved hygiene and perceived improved sexual performance. ${ }^{29}$ Barriers noted in the literature include fear of procedure-related complications and pain, ${ }^{29}$ preference for traditional circumcision by communities that traditionally circumcise, ${ }^{30,31}$ and unfavorable perceptions about the 6-week abstinence from sexual intercourse in the period following circumcision. $^{32}$

Research has also shown that a standard package of core demand creation strategies would not be universally applicable and has underscored the importance of tailoring these strategies to specific country contexts. ${ }^{33}$ According to a recently published study from Zimbabwe, the strongest predictor of VMMC uptake was self-efficacy - where men who have high self-efficacy (one's belief in one's ability to get circumcised) were eight times more likely of having been circumcised than those men with low levels of selfefficacy. ${ }^{29}$

There is a growing body of literature exploring how these cultural norms are interrelated with VMMC uptake, ${ }^{28,30,34-36}$ emphasizing the importance of cultural considerations when developing demand creation strategies for various segments of the population to maximize the VMMC uptake. Some national programs have explored strategies for effectively engaging communities and increasing uptake of VMMC through culturally sensitive mobilization and collaborating with traditional circumcisers. ${ }^{30,37}$

\section{Medical products, vaccines, and technologies}

\section{Successful VMMC implementation requires coordinated partnerships that are effective and efficient in meeting commodity needs}

The implementation of VMMC services requires a complex logistics system, involving commodity procurement, supply chain considerations, human resources, waste management, and warehousing. Factors facilitating VMMC scale-up include the development of a standard list of VMMC commodities as well as the packaging of VMMC surgical kits as an efficiency measure. ${ }^{38}$ The Partnership for Supply Chain Management was instrumental in providing technical and programmatic support on procurement, logistics and waste management to the Government of Swaziland in its effort to implement the Accelerated Saturation Initiative. ${ }^{38}$ The implementation of VMMC programs often involves the creation of a supply chain system that is managed by an implementing partner and is parallel to the supply chain for national essential medicines. ${ }^{38}$ The provision of equipment and supplies from the partner organizations to the facilities was noted as facilitating program delivery in Kenya during the first year of implementation. ${ }^{26}$

In 2012 , ten of the 14 priority countries reported challenges related to inadequate supplies and delayed procurement. ${ }^{5}$ As highlighted in a recent quality assessment, challenges related to commodity procurement may reflect a broader health system strengthening need not unique to VMMC. ${ }^{24}$ In addition to procurement, challenges in waste management also exist. Botswana reported challenges in the waste management of disposable kits during outreach services. ${ }^{5}$ It is important to note that waste management and supply chain costs are often not included in economic evaluations. ${ }^{39}$ An extensive cost evaluation in Swaziland found that supply chain and waste management costs add an additional US $\$ 60$ per VMMC, nearly doubling the cost per procedure reported by many estimates. ${ }^{38}$

The use of nonsurgical devices is a recent technological innovation related to VMMC. These innovative medical devices have been promoted as potential alternatives to conventional surgery and have shown promising results during clinical trials in the areas of safety and acceptability. ${ }^{40,41}$ There is further opportunity for medical devices to both decrease the human resource demands associated with the scale-up through task shifting and increasing operator efficiency, ${ }^{42,43}$ as well as to support current demand creation activities aimed at improving accessibility to VMMC services. The nonsurgical devices are much easier to deploy within 
current clinical settings or during outreach activities as they do not require a sterile environment or the breadth of equipment required for the surgical procedure. However, whether the introduction of these devices in VMMC service delivery will dramatically improve uptake remains to be evaluated.

\section{Health systems financing Continued donor funding is critical for programmatic success}

It was estimated that US \$2 billion would be needed to scale-up VMMC coverage to $80 \%$ in the 14 priority countries. ${ }^{44}$ The availability of external funding has been a major facilitator related to VMMC scale-up. All 14 priority countries have donor-funded partner organizations supporting VMMC activities. ${ }^{5}$ PEPFAR has been the primary funder of VMMC programs in Africa, with a total of US \$250 million dollars having been allocated to VMMC activities through 2011. Also funding the VMMC programs are: the Bill and Melinda Gates Foundation; the Global Fund to Fight AIDS, Tuberculosis, and Malaria; and the World Bank. ${ }^{5}$ Several recent studies have begun examining cost drivers related to VMMC, finding that personnel have a substantial impact on the cost per procedure. ${ }^{27,39}$ Economies of scale analyses indicate that VMMC programs are still at the scale-up phase, with program investment cost and low patient volume contributing to higher costs per procedure. ${ }^{27}$

A useful resource for funding advocacy is the DecisionMakers' Program Planning Tool, a modeling tool developed collaboratively by the United States Agency for International Development (USAID) and UNAIDS. This tool can help decision makers to estimate the epidemiological impact and cost of the alternative programmatic options for scaling-up VMMC. Both Namibia and Botswana used these results to develop and refine national strategies for male circumcision, outlining the target population for the intervention and the pace of the scale-up. ${ }^{45}$ Additionally, the Decision-Makers' Program Planning Tool can be useful in calculating the net savings associated with HIV infections averted as well as subsequent treatment costs averted. ${ }^{44}$

Reliance on donor funding for the scale-up of VMMC services is proving to be a barrier in some priority countries where VMMC achievements have been low. Donors are typically interested in rapid results. When these are not met, donors have abruptly stopped funding VMMC programs (R. Lebelonyane, Ministry of Health, Botswana, personal communication, September 2013), leaving governments who had not budgeted for the massive scale-up stranded and without the financial means to continue the
VMMC efforts. Some countries are avoiding reliance on donor funding by generating and directing national funds specific to HIV programs. South Africa invests a significant amount of national treasury funds into its VMMC program. ${ }^{46}$ Countries that are adopting alternative methods of raising national funds for their HIV programs include Zimbabwe, which charges a 3\% AIDS levy on income, and Uganda, which established a US \$1 billion HIV trust fund. The latter is also expected to generate income through levies on bank transactions, cigarettes, alcoholic beverages, and trading activities. ${ }^{47,48}$ However, information on percentage spending on VMMC from these levies/funds is currently not available.

\section{Health information}

\section{Evidence-based decision making} is critical for achieving VMMC targets

Quality information is needed to guide evidenced-based decisions on how to allocate limited resources for HIV prevention, including the VMMC programs. Specifically, data are needed to determine effectiveness, epidemiological and economic impact, and programmatic performance of VMMC programs. The development of a standardized set of indicators agreed upon by technical ${ }^{49}$ and funding agencies ${ }^{50}$ is one factor that strengthened the monitoring and the evaluation of VMMC services. WHO/UNAIDS developed an indicator guidance document, ${ }^{49}$ which used the same indicators, disaggregation, and definitions as the PEPFAR-revised guidelines. ${ }^{50}$ The latter classifies and lists indicators as essential or recommended, based on the reporting priorities. The number of indicators has been reduced to approximately five, ensuring that the most important components of the program are captured. It also limits the data capture burden on health care providers. ${ }^{50}$ The guidelines also provide mechanisms to ensure data quality, which is an important aspect of health information systems.

Ensuring that the data collected through the national health information systems are of sufficient quality for meaningful interpretation is a challenge in many resourcelimited countries. ${ }^{51}$ Therefore, the VMMC monitoring systems in most of the priority countries are parallel to national health information systems. By 2012, only six of the priority countries reported having $\mathrm{VMMC}$ reporting systems that were integrated into the national monitoring and evaluation systems. ${ }^{5}$ This is particularly unfortunate, given that the data suggest that the introduction of the VMMC services can yield improvements related to the existence of a functioning information system. ${ }^{23}$ 
Reporting to technical and funding agencies has largely focused on the absolute number of eligible men circumcised. Programs often give priority to maintaining information systems that capture the number of circumcision procedures performed due to the pressure from stakeholders to meet targets, as opposed to the information systems that record data to guide service delivery and quality of care. ${ }^{23}$ The data that is needed for on-the-ground program implementation is not the same as the information needed for overall program monitoring. For example, the data related to the seasonal fluctuations in the VMMC demand may not be meaningful at the national level, but that information is important at the local level for staffing plans and demand creation activities. There is a need for tools and systems to help program managers collect data for local decision making, especially in managing supply and demand. ${ }^{6}$ In Tanzania, the live capture and reporting of service delivery data through web-based data collection systems helped inform leadership about trends in service delivery so that the adjustments could be made in a timely manner. ${ }^{20}$

An emphasis is also needed on the monitoring of data related to adverse events. Priority countries have consistently reported adverse event rates to be $<2 \%,{ }^{5}$ compared to the $3.5 \%-3.8 \%$ reported in the controlled clinical trials. ${ }^{2,4}$ The expectation, however, has been for adverse events at the community level to be higher than those of randomized clinical trials. However, assessing complication rates following VMMC has not been standardized, which hampers comparisons. ${ }^{52}$ Several papers have highlighted that systems are unsatisfactory for tracking adverse events. ${ }^{23,24,53}$ Efforts are needed to refine and standardize adverse event reporting mechanisms and to ensure that these data are not underreported for fear of reflecting poorly on a program. ${ }^{23}$

\section{Conclusion}

Male circumcision has been proven to be an effective add-on strategy for HIV prevention in randomized clinical trials ${ }^{2-4}$ and at the community level. ${ }^{54}$ Costing studies have shown that the rapid scale-up of VMMC to achieve the 20 million circumcision targets by 2015 can avert 3.4 million new HIV infections, and save US \$16.5 billion dollars in HIV treatment costs by $2025 .{ }^{44} \mathrm{WHO} /$ UNAIDS set an ambitious goal for the VMMC. The nature of the procedure makes it a challenging intervention to deliver as it involves healthy men, who generally have low health seeking behaviors, ${ }^{55}$ undergoing an elective surgical procedure with cultural, ethnic, political, and religious dimensions. Even though the VMMC programs have grown dramatically since the WHO/UNAIDS recommendations, it is unlikely they will achieve the ambitious goals by 2016 .

This review article has taken a systems thinking approach to examining barriers and facilitators of VMMC uptake. This is a WHO-recommended approach for comprehensively examining how interventions can operate more successfully and effectively in complex, real-world settings. ${ }^{8}$ Each of the six components of the WHO health systems building block framework should be analyzed on a country-by-country basis to better understand steps needed to maximize VMMC uptake in different settings. Several of the critical health systems barriers related to leadership, human, and material resources are gradually being addressed. This is evidenced by the improved VMMC program achievements in priority countries, which have gone from nearly 600,000 in 2010 to 6 million by the end of 2013. ${ }^{5,6}$ Currently, the main barrier to the rapid scale-up of VMMC in the majority of the priority countries is demand creation. Understanding the contextual barriers and facilitators of VMMC for the most at-risk target population in each priority country is important in generating demand. ${ }^{6,56}$ Success is highly dependent on funding being available. ${ }^{6}$ As VMMC programs scale-up, quality of services ${ }^{24}$ and workforce factors ${ }^{22}$ need to be monitored closely.

Countries implementing VMMC programs should share their experiences more broadly and systematically to identify and to enhance best practices as well as to identify and to address barriers to successful VMMC scale-up. The clearinghouse on male circumcision for HIV prevention is a useful resource for policy documents, guidelines, and reports. ${ }^{57}$ Priority countries and implementing partners should be encouraged to post regularly comprehensive program reports and findings from operations research to this site for the purposes of sharing best practices.

\section{Author contributions}

$\mathrm{RN}, \mathrm{JL}$, and BS conceived and designed the manuscript. RN, $\mathrm{JL}, \mathrm{JH}, \mathrm{MM}$, and BS wrote the manuscript. JG participated in the revision of the manuscript. All authors made substantial contributions to conception and design, acquisition of data, or analysis and interpretation of data. All authors critically revised manuscript and approved the final version.

\section{Acknowledgments}

Funding was provided by: PEPFAR to the University of Washington and International Training and Education Center for Health through the US Centers for Disease Control and Prevention, grant number 2GPS001824; through the US Department of Health and Human Services, Health 
Resources and Services Administration Global HIV/AIDS Bureau, Cooperative Agreement \# U91HA06801; and the Afya Bora Consortium Fellowship in Global Health Leadership supported by National Institutes of Health Office of AIDS Research and CDC/PEPFAR, grant \# U91HA06801B.

\section{Disclosure}

The authors report no conflicts of interest in this work.

\section{References}

1. World Health Organization and the Joint United Nations Program on HIV/AIDS. New Data on Male Circumcision and HIV Prevention: Policy and Programme Implications. Geneva: World Health Organization; 2007. Available from: http://libdoc.who.int/ publications/2007/9789241595988_eng.pdf. Accessed April 25, 2014.

2. Auvert B, Taljaard D, Lagarde E, Sobngwi-Tambekou J, Sitta R, Puren A. Randomized, controlled intervention trial of male circumcision for reduction of HIV infection risk: the ANRS 1265 Trial. PLoS Med. 2005;2(11):e298.

3. Bailey RC, Moses S, Parker CB, et al. Male circumcision for HIV prevention in young men in Kisumu, Kenya: a randomised controlled trial. Lancet. 2007;369(9562):643-656.

4. Gray RH, Kigozi G, Serwadda D, et al. Male circumcision for HIV prevention in men in Rakai, Uganda: a randomised trial. Lancet. 2007;369(9562):657-666.

5. World Health Organization, WHO Progress Brief - Voluntary medical male circumcision for HIV prevention in priority countries of East and Southern Africa. July 2014. Available from: http://www.who.int/hiv/ topics/malecircumcision/male-circumcision-info-2014/en/ Accessed May 15, 2014.

6. Sgaier SK, Reed JB, Thomas A, Njeuhmeli E. Achieving the HIV prevention impact of voluntary medical male circumcision: lessons and challenges for managing programs. PLoS Med. 2014;11(5): e1001641.

7. The United States President's Emergency Plan for AIDS Relief [homepage on the Internet]. Tenth Annual Report to Congress on PEPFAR (2014). Treatment: Direct FY2013 Voluntary Male Medical Circumcision Results; 2014 [cited May 22, 2014]. Available from: http:// www.pepfar.gov/press/222833.htm. Accessed May 25, 2014.

8. World Health Organization. Systems Thinking for Health Systems Strengthening. Geneva: World Health Organization; 2009. Available from: http://whqlibdoc.who.int/publications/2009/9789241563895_eng. pdf. Accessed May 15, 2014.

9. World Health Organization. Everybody's Business: Strengthening Health Systems to Improve Health Outcomes. WHO's Framework for Action. Geneva: World Health Organization; 2007. Available from: http://www. who.int/healthsystems/strategy/everybodys_business.pdf. Accessed May 15, 2014.

10. World Health Organization. Progress in scale-up of male circumcision for HIV prevention in Eastern and Southern Africa: Focus on service delivery. Geneva: World Health Organization; 2011. Available from: http://whqlibdoc.who.int/publications/2011/9789241502511_eng. pdf?ua=1. Accessed May 15, 2014.

11. Dickson KE, Tran NT, Samuelson JL, et al. Voluntary medical male circumcision: a framework analysis of policy and program implementation in eastern and southern Africa. PLoS Med. 2011;8(11):e1001133.

12. Government of Kenya. Progress Report On Kenya's Voluntary Medical Male Circumcision Programme, 2008-2010. Nairobi, Kenya: Ministry of Public Health and Sanitation; 2012. Available from: http://www. malecircumcision.org/country_updates/documents/VMMC_2010_ report_final.pdf. Accessed May 15, 2014.
13. Centers for Disease Control and Prevention (CDC). Progress in voluntary medical male circumcision service provision - Kenya, 2008-2011. MMWR Morb Mortal Wkly Rep. 2012;61(47):957-961.

14. Mwandi Z, Murphy A, Reed J, et al. Voluntary medical male circumcision: translating research into the rapid expansion of services in Kenya, 2008-2011. PLoS Med. 2011;8(11):e1001130.

15. Auvert B, Marseille E, Korenromp EL, et al. Estimating the resources needed and savings anticipated from roll-out of adult male circumcision in Sub-Saharan Africa. PLoS One. 2008;3(8):e2679.

16. World Health Organization. Considerations for implementing models for optimizing the volume and efficiency of male circumcision services. Geneva: World Health Organization; 2010. Available from: http://www. malecircumcision.org/programs/documents/mc_MOVE_2010_web.pdf. Accessed May 10, 2014.

17. Mavhu W, Frade S, Yongho AM, et al. Provider Attitudes toward the Voluntary Medical Male Circumcision Scale-Up in Kenya, South Africa, Tanzania and Zimbabwe. PLoS One. 2014;9(5):e82911.

18. Ford N, Chu K, Mills EJ. Mills, Safety of task-shifting for male medical circumcision: a systematic review and meta-analysis. AIDS. 2012;26(5): 559-566.

19. Buwembo DR, Musoke R, Kigozi G, et al. Evaluation of the safety and efficiency of the dorsal slit and sleeve methods of male circumcision provided by physicians and clinical officers in Rakai, Uganda. BJU Int. 2012;109(1):104-108.

20. Curran K, Njeuhmeli E, Mirelman A, et al. Voluntary medical male circumcision: strategies for meeting the human resource needs of scale-up in southern and eastern Africa. PLoS Med. 2011;8(11):e1001129.

21. Lissouba P, Taljaard D, Rech D, et al. A model for the roll-out of comprehensive adult male circumcision services in African low-income settings of high HIV incidence: the ANRS 12126 Bophelo Pele Project. PLoS Med. 2010;7(7):e1000309.

22. Perry L, Rech D, Mavhu W, et al. Work Experience, Job-Fulfillment and Burnout among VMMC Providers in Kenya, South Africa, Tanzania and Zimbabwe. PLoS One. 2014;9(5):e84215.

23. Rech D, Spyrelis A, Frade S, et al. Implications of the fast-evolving scale-up of adult voluntary medical male circumcision for quality of services in South Africa. PLoS One. 2014;9(5):e80577.

24. Jennings L, Bertrand J, Rech D, et al. Quality of Voluntary Medical Male Circumcision Services during Scale-Up: A Comparative Process Evaluation in Kenya, South Africa, Tanzania and Zimbabwe. PLoS One. 2014;9(5):e79524.

25. The United States President's Emergency Plan for AIDS Relief. PEPFAR's Best Practices for Voluntary Medical Male Circumcision Site Operations. Washington, DC: The United States President's Emergency Plan for AIDS Relief; 2012. Available from: http://www.usaid.gov/sites/ default/files/documents/1864/pepfar_best_practice_for_vmmc_site_ operations.pdf. Accessed May 15, 2014.

26. Herman-Roloff A, Llewellyn E, Obiero W, et al. Implementing voluntary medical male circumcision for HIV prevention in Nyanza Province, Kenya: lessons learned during the first year. PLoS One. 2011;6(4):e18299.

27. Bollinger L, Adesina A, Forsythe S, Godbole R, Reuben E, Njeuhmeli E. Cost drivers for voluntary medical male circumcision using primary source data from sub-saharan Africa. PLoS One. 2014; 9(5): 84701.

28. Ashengo TA, Hatzold K, Mahler H, et al. Voluntary Medical Male Circumcision (VMMC) in Tanzania and Zimbabwe: Service Delivery Intensity and Modality and Their Influence on the Age of Clients. PLoS One. 2014;9(5):e83642.

29. Hatzold K, Mavhu W, Jasi P, et al. Barriers and Motivators to Voluntary Medical Male Circumcision Uptake among Different Age Groups of Men in Zimbabwe: Results from a Mixed Methods Study. PLoS One. 2014;9(5):e85051.

30. Wambura M, Mwanga JR, Mosha JF, Mshana G, Mosha F, Changalucha J. Acceptability of medical male circumcision in the traditionally circumcising communities in Northern Tanzania. BMC Public Health. 2011;11:373. 
31. Mshana G, Wambura M, Mwanga J, Mosha J, Mosha F, Changalucha J. Traditional male circumcision practices among the Kurya of North-eastern Tanzania and implications for national programmes. AIDS Care.

32. Herman-Roloff A, Otieno N, Agot K, Ndinya-Achola J, Bailey RC. Acceptability of medical male circumcision among uncircumcised men program. PLoS One. 2011;6(5):e19814.

33. Bertrand JT, Njeuhmeli E, Forsythe S, Mattison SK, Mahler H, Hankins CA. Voluntary medical male circumcision: a qualitative study exploring the challenges of costing demand creation in eastern and southern Africa. PLoS One. 2011;6(11):e27562.

34. Plotkin M, Castor D, Mziray H, et al. "Man, what took you so long?" Social and individual factors affecting adult attendance at voluntary medical male circumcision services in Tanzania. Global Health: Science and Practice. 2013;1(1):108-116.

35. Macintyre K, Andrinopoulos K, Moses N, et al. Attitudes, Perceptions and Potential Uptake of Male Circumcision among Older Men in Turkana County, Kenya Using Qualitative Methods. PLoS One. 2014;9(5):e83998.

36. Westercamp M, Agot KE, Ndinya-Achola J, Bailey RC. Circumcision preference among women and uncircumcised men prior to scale-up of male circumcision for HIV prevention in Kisumu, Kenya. AIDS Care. 2012;24(2):157-166.

37. Peltzer K, Kanta X, Banyini M. Evaluation of a safer male circumcision training programme for Ndebele traditional surgeons and nurses in Gauteng, South Africa: using direct observation of circumcision procedures. Afr J Tradit Complement Altern Med. 2009;7(2):153-159.

38. Edgil D, Stankard P, Forsythe S, et al. Voluntary medical male circumcision: logistics, commodities, and waste management requirements for scale-up of services. PLoS Med. 2011;8(11):e1001128.

39. Menon V, Gold E, Godbole R, et al. Costs and Impacts of Scaling up Voluntary Medical Male Circumcision in Tanzania. PLoS One. 2014;9(5):e83925

40. Reed JB, Njeuhmeli E, Thomas AG, et al. Voluntary medical male circumcision: an HIV prevention priority for PEPFAR. JAcquir Immune Defic Syndr. 2012;60 Suppl 3:S88-S95.

41. Sokal DC, Li PS, Zulu R, et al. Randomized controlled trial of the shang ring versus conventional surgical techniques for adult male circumcision: safety and acceptability. J Acquir Immune Defic Syndr. 2014;65(4):447-455.

42. Mutabazi V, Kaplan SA, Rwamasirabo E, et al. One-arm, open-label, prospective, cohort field study to assess the safety and efficacy of the PrePex device for scale-up of nonsurgical circumcision when performed by nurses in resource-limited settings for HIV prevention. $J$ Acquir Immune Defic Syndr. 2013;63(3):315-322.

43. Duffy K, Galukande M, Wooding N, Dea M, Coutinho A. Reach and cost-effectiveness of the PrePex device for safe male circumcision in Uganda. PLoS One. 2013;8(5):e63134.

44. Njeuhmeli E, Forsythe S, Reed J, et al. Voluntary medical male circumcision: modeling the impact and cost of expanding male circumcision for HIV prevention in eastern and southern Africa. PLoS Med. 2011;8(11):e1001132.

45. United Nations Agency Program on HIV/AIDS. Using the DecisionMakers' Programme Planning Tool for Male Circumcision Service Scale-up. Meeting Report. Geneva: World Health Organization; 2008. Available from: http://www.unaids.org/en/media/unaids/contentassets/ dataimport/pub/report/2009/jc1699_dakar_meeting_report_en.pdf. 2011;23(9):1111-1116. in Kenya one year after the launch of the national male circumcision Accessed May 15, 2014.

46. Pillay Y. South Africa Innovates to Scale up and Sustain its HVI Response. Johannesburg, South Africa: Department of Health; 2012. Available from: http://www.unaids.org/en/media/unaids/contentassets/ documents/pcb/2012/PCB31_presentation_SouthAfricaInvestment_ en.pdf. Accessed May 15, 2014.

47. United States Agency for International Development. Technical Brief: Voluntary Male Medical Circumcision (VMMC) for HIV Prevention. Washington, DC: United States Agency for International Development; 2012. Available from: https://www.msh.org/sites/msh.org/files/2012 dec_vmmc_brief_email.pdf. Accessed May 15, 2014.

48. United Nations Agency Program on HIV/AIDS. Efficient and Sustainable HIV Responses: Case Studies on Country Progress. Geneva: Joint United Nations Program on HIV/AIDs; 2013. Available from: http://www.unaids.org/en/media/unaids/contentassets/documents/ unaidspublication/2013/JC2450_case-studies-country-progress_en.pdf. Accessed May 15, 2014.

49. World Health Organization. A Guide to Indicators for Male Circumcision Programmes in the Formal Health Care System. Geneva: World Health Organization; 2009. Available from: http://whqlibdoc.who.int/ publications/2009/9789241598262_eng.pdf. Accessed May 15, 2014.

50. The United States President's Emergency Plan for AIDS Relief (PEPFAR). PEPFAR Guide to Monitoring and Reporting Voluntary Medical Male Circumcision (VMMC) Indicators. Washington, DC: The United States President's Emergency Plan for AIDS Relief; 2013. Available from: http://www.malecircumcision.org/resources/documents/ PEPFAR_guide_to_monitoring_and_reporting_VMMC_indicators_main.pdf. Accessed May 15, 2014.

51. Ledikwe JH, Grignon J, Lebelonyane R, et al. Improving the quality of health information: a qualitative assessment of data management and reporting systems in Botswana. Health Res Policy Syst. 2014;12:7.

52. Kigozi G, Gray RH, Wawer MJ, et al. The safety of adult male circumcision in HIV-infected and uninfected men in Rakai, Uganda. PLoS Med. 2008;5(6):e116.

53. Bertrand JT, Rech D, Omondi Aduda D, et al. Systematic monitoring of voluntary medical male circumcision scale-up: adoption of efficiency elements in kenya, South Africa, Tanzania, and zimbabwe. PLoS One. 2014;9(5):e82518.

54. Auvert B, Taljaard D, Rech D, et al. Association of the ANRS-12126 male circumcision project with HIV levels among men in a South African township: evaluation of effectiveness using cross-sectional surveys. PLoS Med. 2013;10(9):e1001509.

55. Galdas PM, Cheater F, Marshall P. Men and health help-seeking behaviour: literature review. J Adv Nurs. 2005;49(6):616-623.

56. Ssekubugu R, Leontsini E, Wawer MJ, et al. Contextual barriers and motivators to adult male medical circumcision in Rakai, Uganda. Qual Health Res. 2013;23(6):795-804.

57. Family Health International 360 Clearinghouse on Male Circumcision [homepage on the Internet]. Male Circumcision for HIV Prevention. Durham, NC, USA: Clearinghouse on Male Circumcision; 2014. Available from: http://www.malecircumcision.org. Accessed May 15, 2014.

\section{Dovepress}

\section{Publish your work in this journal}

HIV/AIDS - Research and Palliative Care is an international, peerreviewed open-access journal focusing on advances in research in HIV, its clinical progression and management options including antiviral treatment, palliative care and public healthcare policies to control viral spread. The journal welcomes original research, basic science, clinical \& epidemiological studies, reviews \& evaluations, expert opinion \& commentary, case reports \& extended reports. The manuscript management system is completely online and includes a very quick and fair peer-review system. Visit http://www.dovepress.com/ testimonials.php to read real quotes from published authors. 